\title{
Discussion of the Papers Presented at the Symposium
}

\section{Discussion of the paper by ZENSUS (p. 7)}

Morimoto: What roughly is the velocity in the majority of sources?

Zensus: Most sources have apparent velocities between $\sim 2 c$ and $\sim 10 c$.

Schilizzi: What limits can you place on interblob emission in superluminal sources? Are these sources really jets or a sequence of bullets or plasmons being fired out from the nucleus?

Biretta: In 3C345, the region between the superluminal blobs is roughly ten times fainter than the knots, or hundred times fainter than the core.

Discussion of the paper by BARTHEL (p. 17)

Porcas: How well aligned are the mas and arcsec jet in 4C34.37? The alignment/misalignment statistics give another constraint on how to adjust the "standard" model.

Barthel: They are misaligned by $5^{\circ}$. I agree. Naively, one would expect beaming effects to be dominant in the plane defined by mas jet and observer, which would result in the generally observed alignment.

Preuss: With regard to core prominence in the large double-lobed quasars you mentioned, I would like to point out that the largest radio galaxies known also tend to have relatively strong cores.

Barthel: I am fully aware of that but did not want to include radio galaxies in the present discussion since on average radio galaxy cores are one order of magnitude less luminous than those in quasars. Particularly interesting in this respect is the giant radio galaxy $3 \mathrm{C236}$, the largest known object in the universe. We are currently investigating the presence of possible superluminal motion in its core.

\section{Discussion of the paper by BIRETTA (p. 19)}

Ulvestad: When comparing the internal pressure of superluminal components with external pressure in the narrow line region, you should be careful about the pressure assumed for the narrow line region. In nearby active galaxies, there is some evidence for stratification in density in the narrow line region. Therefore, 
the density and pressure of that region within a few parsecs of the core may be considerably higher than in the material hundreds of parsecs from the core.

Biretta: We simply used an upper limit to the NLR density of $10^{6} \mathrm{~cm}^{-3}$ in computing the external pressures and compared this to knots 20 to $200 \mathrm{pc}$ from the nucleus. A more detailed model for the NLR densities that includes stratification would be useful.

Chu: Have you any intention to make the VLBI polarization observations so as to confirm the magnitude of magnetic fields?

Biretta: Others are doing this.

Marcaide: Do you have any reason to believe that the core position shift with frequency should not also be found in other superluminals? Note: This shift was first found for $1038+528 \mathrm{~A}$ with $3.6 / 13 \mathrm{~cm}$ observations and a proper registration, leading to the result that $1038+528 \mathrm{~A}$ is a mild superluminal.

Biretta: No. It seems likely that similar shifts will be found in other superluminals, as a similar shift was found (by you) in the quasar pair $1038+528$ using phase reference VLBI.

R. J. Davis: Are you worried by the chance that the initial orientation is $1^{\circ}$ to the line of sight?

Biretta: This is a question of statistics. It would be worrisome if many sources required an alignment within $1^{\circ}$ of the line of sight, but it is not a serious problem in any one source. Further, if $\mathrm{C4}$ has an intrinsic acceleration, the initial angle to the line of sight could be much larger than $1^{\circ}$.

Niell: If you calculate the speed of the components along the track (arclength), wouldn't the acceleration be significantly larger? (Upon further thought: If components approach an asymptotic direction, then accounting for arclength will reduce the increase of $v / c$.)

Biretta: The motion of $\mathrm{C} 4$ is initially nonradial and later becomes nearly radial. Computing the proper motion along the path would give a slightly larger initial speed and slightly less acceleration than when only the radial component of motion is considered.

vanBreugel: You mentioned two possible explanations for the curvature observed in 3C345: bending by external gas or internal jet stabilities. Could the curvature also not be due to precession of the nucleus?

Biretta: Precession cannot work in its simplest form since then the superluminal expansion would not be constant.

vanBreugel: But don't you think that there could be an external medium in addition to precession?

Biretta: Yes, but then any precession model could work. 
vanBreugel: That was exactly my point.

Shaffer: How confident are you that the apparent shift in position of the core (with frequency) is not due to optical depth effects in the jet components?

Biretta: It is possible that the position shifts occur in the knots. However, the shifts are about $1 / 3$ of the knot diameters, so that very strong spectral index gradients would be required.

Barthel: Was the acceleration of $\mathrm{C} 4$ accompanied by component brightening?

Biretta: Yes. The acceleration occurred near epoch 1982, and the flux of C4 doubled between 1981 and 1983 . The acceleration and brightening might be related kinematically, though the flux of $\mathrm{C4}$ will be affected by intrinsic evolution as well as by kinetic changes.

\section{Discussion of the paper by $\underline{\operatorname{SIMON}}$ (p. 21)}

Porcas: How colinear are the three VLBI components?

Simon: The three VLBI components are colinear to within the measurement errors. The stationary component is at $P A=118^{\circ} \pm 1$, while the superluminal component is at $117^{\circ} \pm 4$. The arcsecond component is at $P A \sim-50^{\circ}$, within 8 degrees of the axis defined by the VLBI components.

Barthel: Since the superluminal motion relies heavily on the $1979.8 \mathrm{map}$, is it possible that you missed the moving component at that epoch?

Simon: No. The superluminal component was clearly seen in the 1979.8 map and in each of the subsequent maps. It has faded somewhat with time, but that has been compensated for by the recent maps having slightly higher dynamic range.

Cohen: Superluminal motion always is jittery, and a slowdown is expected here if a new component is coming out of the core. You measure from the centroid of (core + new component).

Simon: This might explain these observations, but the discrepancy is larger than a beam diameter. There is no evidence of any extension to the core away from the superluminal component, as might be expected if the change in speed is a resolution effect.

Cotton: If the apparent superluminal motion decreased by about 10 , then the apparent brightness of that component should have decreased by a factor of about 1000. Doesn't this cause difficulty to the interpretation of this component?

Simon: Yes, if the moving component were assumed to be a simple plasmoid. Obviously, the moving component in 3C395 is more complicated than that. A random theoretician could probably cobble together at least two models before breakfast; more observations are clearly needed. 
Discussion of the paper by SALVATI (p. 47)

deBruyn: Although the x-ray intensity in 3 C273 fits the SSC model predictions, its slope certainly doesn't. Doesn't this worry you?

Salvati: The local slope in any given spectral band depends on the electron energy distribution. This is treated very crudely in the present version of the model; in fact, it predicts monoenergetic electrons and very bumpy synthetic spectra. Until the electron energy distribution is treated in a more realistic way, the model will only give a global slope over several spectral bands.

Biermann: For our Bonn sample of flat spectrum radio sources, we have observed all sources with the $30-\mathrm{m} \mathrm{mm}$-telescope at a wavelength of $1 \mathrm{~mm}$. All sources have a flat or nearly flat spectrum to $1 \mathrm{~mm}$. One source, the BL Lac $1803+78$, still has nearly $1 \mathrm{Jy}$ at $60 \mu$ (IRAS). Thus, we confirm that the turnover in the overall spectrum is near $10^{12} \mathrm{~Hz}$, consistent with your picture.

In your initial expression for the total luminosity, the Klein-Nishina cutoff limits the Compton orders to about 2 at most. From the mm-x-ray correlation, the Lorentz factor can be estimated to about $10^{3}$, limiting you indeed to 2 orders.

Salvati: The Lorentz factor can be lower and the number of Compton orders larger if one allows for an intermediate order between submm and x-ray wavelengths. Obviously, very low Lorentz factors are ruled out by synchrotron opacity limits.

\section{Discussion of the paper by $\underline{\text { LIND }}$ (p. 55)}

vanBreugel: Given the many different parameters that can determine the observed flux density variabilities and velocities in "real" jets (shocks, obstacles, opacity effects, viewing angles, etc.), isn't it surprising that the superluminal expansion speeds that are observed seem to be rather constant in time? Could this not be used to constrain your "more realistic" models?

Lind: The constancy for an individual component would provide some limit on the evolution of patterns within a jet, e.g., shocks do not slow down appreciably. Similarities between a number of components within a given source suggest that the ejection of the brightest features is fairly uniform in time. So, constancy of one component indicates limited component evolution; constancy of characteristics of different components indicates limited central engine evolution and/or precession, over time scales of years.

\section{Discussion of the paper by $\underline{\text { READHEAD }}$ (p. 65)}

Wilkinson: Now that MERLIN has been enhanced with the link into Cambridge, one can observe at a low frequency, $408 \mathrm{MHz}$, with a resolution of $\sim 0.5$ arcsec. It may well be that one will be able to map the weak, steep-spectrum, extended emission in the very core-dominated objects in your sample that are impossible to map with the VLA at $\sim 5 \mathrm{GHz}$. This would help your statistics on the coreextended structure position angle differences. 
Readhead: This would be well worth doing. It could almost double the number of objects that we could include in our misalignment analysis.

Porcas: Istvan Fejes and I have EVN data at $\lambda 18 \mathrm{~cm}$ and $\lambda 50 \mathrm{~cm}$ that indicate that the VLBI jet turns anticlockwise toward the direction of the southwest arcsecond scale component.

Readhead: Thank you. We shall use this result in calculating the misalignment in this object.

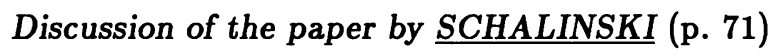

vanBreugel: Of the four objects without redshifts, are these highly polarized? Are they BL Lac objects?

Schalinski: Polarization observations, both at optical and radio frequencies, are consistent with the identification of the four sources as BL Lac type objects.

Discussion of the paper by MUTEL (p. 73)

Lind: Since the momentum transfer surface between the jet and the surrounding medium can be considerably wider than the jet or the terminal hot-spot (if the terminal hot-spot corresponds to the terminal mach disk in numerical simulations), then one can have considerably slower velocities of expansion than estimated in the ram-pressure calculations.

Porcas: Have you managed to find any central components yet?

Mutel: No!

Neff: What are the optical properties of the galaxies in which the CD's are found? You mentioned that all CD's were in galaxies or EF's, not QSO's, but there are different sorts of galaxies. What are the infrared properties of the systems? It would be useful to know if the optical spectra show evidence for an AGN (BLR, outflow, etc.).

Mutel: In general, not much is known about the optical properties of CD sources, except that the few galactic identifications appear to be elliptical. I have checked the IRAS point source catalog for all known CD sources. There are no CD sources listed.

Preuss: With regard to the evolutionary sequence you propose: Do the statistics of the source types you consider as evolutionary stages agree with what you would expect from the characteristic time scales?

Mutel: The relative number of CD, SSC, and FRII doubles at a given luminosity depends greatly on the time dependence of the expansion speed and the luminosity evolution, neither of which are known. 
Discussion of the paper by MARR (p. 91)

Shapiro: Can you comment on the apparent change in orientation of the "symmetry axis" of the core from $P A \sim 30^{\circ}$ in 1981 (April and December) to $P A \sim 0^{\circ}$ in $1984-85$ ?

Marr: The core is the dominant component in our maps at $1.3 \mathrm{~cm}$ and at $2.8 \mathrm{~cm}$. The structure in this region is consistent) in 1981 at both wavelengths. Unfortunately, the time interval between our images is insufficient to follow the evolution of the core accurately. We have no physical explanation for these structural changes. Precession, or curved paths of particles ejected from the core, are possibilities. We plan to study the core structure in more detail in the future.

\section{Discussion of the paper by WRIGHT (p. 93)}

Biermann: The mm-x-ray connection suggests one more strong argument for mmVLBI. With mm-VLBI and $x$-ray observations we will be able to test all the nice SSC models we discussed this morning. The German x-ray satellite ROSAT is scheduled now to go up about 1990. Germany has just finished a report on the future of astronomy (15 years). In this report, mm-VLBI gets very strong support as a national goal. So we hope to equip the European mm-dishes with VLBI equipment, increasing the sensitivity dramatically.

Wright: Unpublished Einstein data provides evidence for a $30 \%$ to $50 \%$ increase in the x-ray intensity between February 1979 and February 1980, suggesting inverse Compton emission at the time of the millimeter flare. This is consistent with the observed size of the core of $3 \mathrm{C} 84$ at $\lambda 3 \mathrm{~mm}$. We can look forward to a world array at $\lambda 3 \mathrm{~mm}$.

Porcas: How confident are you in the reality of your detection of the 10 mas knot component?

Wright: All the components in the model fit are present in the uncalibrated visibility data. There is evidence for compact structure $\sim 10$ mas to the south of the core of $3 \mathrm{C} 84$ at all epochs. Model fitting determines the flux and location of this component.

Langston: Can you use your proposed multifrequency observations to trace atmospheric phase variations and then lengthen to coherent integration time?

Wright: Observations at three frequencies across the $\lambda 3 \mathrm{~mm}$ window would not be simultaneous and cannot be used to increase the coherent integration time. On the other hand, we do have simultaneous observations at $5 \mathrm{GHz}$ that might be used to track atmospheric fluctuations and provide a phase reference to extend the coherent integration at $\lambda 3 \mathrm{~mm}$.

Preuss: You expressed your confidence in the "bright future" of mm-VLBI. How bright is it actually going to be within, say 5-10 years from now, in terms of sensitivity and image quality? 
Wright: We have demonstrated that there are no major technical problems with VLBI at $\lambda 3 \mathrm{~mm}$. With rather modest improvements in sensitivity and a few additional antennas, we should be able to make images with dynamic ranges of $\sim 10$. Use of the larger telescopes at Nobeyama, Onsala, and IRAM will improve the sensitivity and resolution. The MkIII density upgrade and the use of VLBA antennas and recorders will allow more frequent sampling at higher bandwidth so that in 5-10 years we can expect to see high quality maps with a flux limit $<\sim 100 \mathrm{mJy}$.

\section{Discussion of the paper by FANTI (p. 111)}

Menon: I had shown in the Bologna Symposium Proceedings that for quasars the low frequency turnover suggests that the linear size of compact sources depends on redshift. Is it possible that the size difference between galaxies and quasars that you find is also partly at least a redshift effect since the quasars are in general at higher $z$ than galaxies?

Fanti: The difference we find between galaxies and QSS's is mainly in their morphology. I think the redshift has little relevance since CSS radio galaxies and CSS QSS's in our sample have rather similar redshifts.

\section{Discussion of the paper by VAN BREUGEL (p. 115)}

Birkinshaw: Roger Davies and I have been using the KPNO 4-m telescope to image a large sample of radio galaxies and a companion sample of nonradio galaxies. There are no obvious, dramatic differences between the samples. The strangest galaxies so far are nonradio emitting.

vanBreugel: When discussing galaxy peculiarities, it is important to specify at which level, and of what kind (tidal tails, dust lanes, etc.), they are. Furthermore, (radio) galaxy peculiarities seem to depend on radio power and redshift. For example, the peculiarities found by Heckman et al. (Ap.J.) and Hutchings (1987, preprint) are at a much higher surface brightness level than in the lower luminosity radio galaxies. Also, at very high redshifts, galaxies have peculiarities at even higher surface brightness levels (e.g., McCarthy et al. 1987).

Barthel: In high $z$ quasars where we can study the associated $\left(\Delta v \lesssim 3000 \mathrm{~km} \mathrm{~s}^{-1}\right)$ CIV absorption, it has been found that the occurrence of such systems in steep spectrum QSR's is much higher than in flat spectrum QSR's (Foltz et al., Ap.J., 1986; Barthel et al., in preparation). Such differing environmental properties argue against the unified scheme.

vanBreugel: History shows that ignoring the importance of one's environment can be hazardous.

Wrobel: You seem to want to link optical peculiarities with the presence of powerful radio sources. Have you looked for such peculiarities in radio-quiet ellipticals? 
vanBreugel: A large optical imaging survey of radio-quiet ellipticals has been made recently by Djorgovski (1986, thesis, UC, Berkeley). Surface photometry was obtained of several hundred bright ellipticals. Very few showed evidence for dramatic peculiarities at the same surface brightness levels as found by Heckman et al. (1986). (See also my answer to Dr. Birkinshaw.)

Discussion of the paper by $\underline{B \AA \AA T H}$ (p. 117)

Jauncey: Are you monitoring the $930 \mathrm{MHz}$ flux density history of your sample sources, as it would seem that such close monitoring should be an essential part of your program?

Bååth: The flux densities were measured at Arecibo during the VLBI observations only. The flux density history of these sources is monitored by other groups at nearby frequencies, though.

vanBreugel: What are the optical properties of the sources in your sample?

Bååth: The sources were selected for low frequency variability and in a declination range suitable for Arecibo $\left(\sim 20^{\circ}\right)$. Many of the sources have not been optically identified and thus have no known redshift.

Cohen: Could you comment on the possibility that some variety of interstellar scattering is responsible for the expansions?

Bååth: The components are moving linearly with the same position angle at all three epochs. Scattering should cause the components to wander around, shifting the position angle. Also, the jet in CTA102 seen with the VLA (R. Rusk, this meeting) has a position angle in excellent agreement with the direction of the motion of the component we see in our VLBI maps.

Discussion of the paper by MUXLOW (p. 131)

Romney: Did you find it necessary, or even possible, to apply baseline-based editing in producing images from your large VLBI arrays? VLBI observations are approaching the multiplicity of the VLA, where this sort of processing is generally regarded as impractical.

Muxlow: Both global and baseline-related editing are necessary and were indeed performed on these data. Software development to improve the response time for baseline-related editing is underway.

Discussion of the paper by WARDLE (p. 143)

Pooley: With what accuracy can you place the polarization maps relative to the total intensity?

Wardle: The antenna phases needed to make the polarization map are the same 
antenna phases found by the hybrid-mapping procedure for the total intensity map. To the extent that these phases are determined correctly, the maps are perfectly registered. In practice, we find that the maps are registered to better than 0.2 mas.

deBruyn: The region that is most likely to contribute most to the Faraday depolarization is the transition zone between the BLR and the NLR. We know little about this transition region. Can your data set any limits on the product of $n_{e} B$ in this region?

Wardle: The limit depends on the size and distribution of the clouds, which we do not know. Values of $n_{e} B$ in the range 0.1 to $10 \mathrm{~cm}^{-3}$ seem reasonable.

Inoue: I would like to comment on the penetration of polarized emission. Last year we found a synchronous variation in polarization angle between optical and radio frequencies in $0 J 287$. They varied more than $90^{\circ}$ within a week. So, at least at that time, both polarized regions are associated with each other.

Wardle: Other sources also exhibit episodes where the optical and radio polarizations change together. VLBI polarimetry will be able to determine the location of the varying region (i.e., in the jet or in the core).

Ekers: A smooth and uniformly polarized jet that has a changing position angle of polarized emission can appear double in a barely resolved polarized intensity image (beam depolarization). Consequently, it is unlikely that the solution for the separation of "apparent" components will be unique and capable of giving reliable estimates of component motion.

Wardle: A uniformly polarized jet with a changing position angle can be distinguished from a point double in the $u-v$ plane. We do our model fitting in the u-v plane.

\section{Discussion of the paper by $\underline{R E I D}$ (p. 169)}

Rickett: Carl Gwinn showed earlier that the position errors due to refractive effects in the ISM are (surprisingly) small, $<20 \mu$ as. Evidently, you have considered this as a source of error in your distance estimate to Sgr B2. I would like to point out that if the ISM has a "steep spectrum vs. wavenumber" (as opposed to the "Kolmogorov" spectrum), there is an additional source of error in the proper motion vectors. Have you considered this?

Reid: The limits quoted by Gwinn are from the Sgr B2 data themselves. As such, they place a limit on refractively induced apparent motion about $<20 \mu$ as $\mathrm{yr}^{-1}$ or $<1 \mathrm{~km} \mathrm{~s}^{-1}$. Perhaps with a very steep fluctuation spectrum and a certain pattern speed, the $15 \mathrm{~km} \mathrm{~s}^{-1}$ "turbulent" velocity residuals could be explained by refractive effects. However, even if this were the case, the distance estimate comes from the systematic motions (expansion) and would not be biased in any way. 
Discussion of the paper by BARTEL (p. 175)

Kylafis: 1) If the expanding shell is spherically symmetric, how do you explain the observed polarization? 2) Electron scattering in an expanding shell shifts the spectral lines and gives you a false reading of the expansion velocity. Does that affect your results?

Bartel: 1) Since we have not yet mapped any supernova, we allow, in our estimates of uncertainties, for a (moderate) deviation from spherical symmetry. However, I do not know of any detection of polarized emission of the supernovae I discussed here. 2) Computations of models with electron scattering in the atmosphere show that mostly the shape of the P-Cyg line profiles are changed but hardly so the values for the expansion velocity of the photosphere and for the maximum expansion velocity. Therefore, electron scattering is unlikely to significantly affect our results.

Chu: The value of $H_{0}$ that Cohen inferred from superluminal movement measurements is likely to be $100 \mathrm{~km} \mathrm{~s}^{-1} \mathrm{Mpc}^{-1}$, while from your direct measurements it is $65 \mathrm{~km} \mathrm{~s}^{-1} \mathrm{Mpc}^{-1}$. I would like to know which value of $H_{0}$ is more accurate or more believable.

Bartel: Since the inference of $H_{0}$ from superluminal sources does not (yet) include an estimate of the uncertainty, it is hard to say which of the two values is more accurate. As to the second part of your question, I tend to regard, in general, direct measurements as more believable.

Pacini: I feel that it is dangerous to infer from the radio portion of SN1987A that the radiosphere always expands faster than the photosphere. The emission of SN1947A was a minor prompt event taking place outside the photosphere. The emission of radio supernovae like SN1979C occurs one year or so later, and it may be due to different causes and involve different physical processes.

Bartel: Alternatively, the delay may be due to a circumstellar medium much denser than that of SN1987A. The radio light curve and the evolution of the radio spectrum of SN1987A are very similar to the light curves and spectral evolutions of other supernova; only the time between the supernova explosion and the onset of radio emission is much shorter and the radio luminosity much weaker. I think that it is fair to say that our VLBI results on SN1987A are consistent with our assumptions for other supernova about the location of the radiosphere with respect to the photosphere.

\section{Discussion of the paper by WILKINSON (p. 187) (presented by DeBruyn)}

Molnar: Have the historic plates from the 1940's been searched for an optical supernova?

deBruyn: As far as I know, they have not. We have not taken any action yet but will, in a paper soon to be submitted, draw attention to this possibility.

Pacini: What is the minimum energy content of the SNR? 
deBruyn: It is very comparable to that of most young galactic SNR's, but because of the ten times smaller linear size, it radiates much more efficiently.

Discussion of the paper by TURNER (p. 191)

Rogers: Wouldn't the microlensing or "Einstein" lensing of objects within our galaxy be a very transient phenomenon owing to differential galactic rotation?

Turner: Yes, there would be a transient signature. I haven't thought about this yet. (Answer from Art Niell: About 8 milliarcseconds/year.)

vanBreugel: For an assumed range of $H_{0}=50-100 \mathrm{~km} \mathrm{~s}^{-1} \mathrm{Mpc}^{-1}$, what would the expected time delay be between the gravitationally lensed images in $0957+561$ ?

Turner: The answer is somewhat model-dependent, but the best current estimate is one to two years, with longer delays corresponding to smaller $H_{0}$ values.

Gwinn: 1) Why is high dynamic range necessary to observe gravitational lensing of masers by stars? 2) Does the brighter image remain nearly undeflected?

Turner: 1) It is not strictly necessary, but it allows one to detect large impact parameter (i.e., poor alignment) lensing events. These are the most probable, of course. 2) Yes.

Kylafis: What is the typical time delay for two images if the gravitational lens is a star?

Turner: They are of order of the light travel time across the Schwarzschild radius for a black hole with the mass of the lensing object, i.e., $\sim 10^{-5}$ sec for a solar mass object.

Burke: We have been following the flux variations in $0957+561$ for the past six years using the VLA. We have a result, but it is not yet sufficiently accurate to give a cosmologically interesting answer. The time delay is probably in the range 400-700 days, with A varying first. If B were to vary first, the time delay would have to be greater than six years, although this case is not generally predicted in current models, which favor A varying first.

Discussion of the paper by GORENSTEIN (p. 201)

Backer: On what time scale would you expect changes in the VLBI structure from transverse motion of the line of sight with respect to the lensing system (velocities up to $\left.500 \mathrm{~km} \mathrm{~s}^{-1}\right)$ ?

Gorenstein: Transverse motions yielding apparent displacements of about $0.1^{\prime \prime}$ or more will yield detectable changes in image magnification. This angular scale is set by the $\sim 1^{\prime \prime}$ size of the lensing galaxy. Transverse velocities of $\sim 500 \mathrm{~km} \mathrm{~s}^{-1}$ yield $0.1^{\prime \prime}$ displacements in about $10^{6}$ years at distances of $10^{10}$ lyr. 
Discussion of the paper by BRIGGS (p. 211)

Flatters: Would it be possible to use these techniques to investigate gas close to the quasar itself?

Briggs: Perhaps. High column densities of cool neutral gas $\left(\gtrsim 10^{20} \mathrm{H} / \mathrm{cm}^{2}\right)$ are necessary to create a detectable 21-cm absorption line. If the HI spin temperature is raised, either by collision in a hot gas or by bathing the gas in the 21-cm continuum of a nearby quasar, the optical depth will be reduced. One certainly does not expect 21-cm absorption by the quasar emission line region, for instance.

Neff: 1) Do the 21-cm absorption lines change with time? 2) Do you see absorption lines in the optical spectrum? If so, are they at the same redshift as the HI?

Briggs: 1) Two spectra have been obtained at Arecibo about a year apart. Within the errors, they are consistent with no change. 2) There is a rich optical absorption spectrum at the same redshift. In fact, we selected this object for study at radio wavelengths on that basis (see Wolfe et al. 1985, Ap. J. Lett., 294, L67).

Discussion of the paper by KYLAFIS (p. 223)

Norris: Have you yet had a chance to look at the pumping of the $12-\mathrm{GHz}$ methanol masers?

Kylafis: No, not yet. So far, I have concentrated on water masers.

Reid: You asked why no beautiful SiO maser maps have been made. Basically, we do not have the critical short VLBI baselines at the high frequencies of the SiO transition. The VLBA will help alleviate this deficiency.

Discussion of the paper by HASCHICK (p. 233)

Shaffer: How well do you know that the $\mathrm{H}_{2} \mathrm{O}$ maser region is coincident with the nucleus of the galaxy? How well are relative optical, radio continuum, and $\mathrm{H}_{2} \mathrm{O}$ positions known?

Haschick: The optical positions are good to a few arcseconds. The radio continuum and $\mathrm{H}_{2} \mathrm{O}$ positions are coincident to within 0"'05.

Claussen: NGC1068 has a nuclear radio continuum triple source. The water masers fall on top of part of that triple. The radio continuum towards NGC4258 shows only one weak continuum source. The maser source falls on top of it.

Discussion of the paper by LESTRADE (p. 265)

Spencer: Could the decrease in the size of Algol with frequency as measured with VLBI be due to interstellar scattering rather than an intrinsic effect? 
Lestrade: The distance to Algol is 27 parsecs and so interstellar scattering is very small and would not broaden the radio image of Algol.

Kaufmann: I have comments that are somewhat independent of your very interesting talk. It might be dangerous to make a direct physical association between solar and stellar activities. Sunspots hardly affect a fraction of a percent of solar irradiance, while "starspots" must be huge and of entirely different nature (if they really exist). Gyrosynchrotron masering produces very fast pulses in the Sun (milliseconds), while flares in stars are considerably longer. I finally wish to suggest VLBI experiments on the closest star, the Sun. The physics of active regions and flaring processes would greatly benefit from this high spatial resolution diagnostics.

Lestrade: I agree that the solar analogy must be used with care and that the stars that are $10^{4}$ to $10^{5}$ more radio luminous than the Sun might involve different rather than scaled-up emission mechanisms. This cyclotron masering is a short time scale phenomenon (millisecond), but multiple masers might occur and last much longer, as suggested by our observations.

Preuss: The registration of the radio image of UX Ari with the optical star looks plausible. Do you consider that objects of this type are particularly promising candidates for tying radio and optical reference frames together?

Lestrade: Actually, our VLBI project is motivated by the potential of VLBI observations of radio stars to tie astrometrically the optical and radio reference frames. The coincidence of the optical and radio components of the RS CVn binaries is an important question that should be answered by tackling the astrophysical problem of understanding the radio emission mechanism in these binaries.

\section{Discussion of the paper by VERMEULEN (p. 275)}

Biretta: In your beautiful sequence of maps, the radio emitting knots are ejected simultaneously in opposite directions. Is the ejection always simultaneous in the two directions?

Vermeulen: These maps indeed show blobs being ejected simultaneously to the east and to the west. No other such sequence of hybrid maps exists, but model fitting to an earlier sequence of observations by Schilizzi et al. (IAU Symposium 110, 1984, p. 280) indicated rather one-sided emission.

Ball: As a rule, one cannot do earth-rotation interferometry on an object that changes significantly with respect to the synthesized beamwidth in a day's time. This would seem to apply to SS443. Are your maps perhaps confused by this effect?

Vermeulen: We are fully aware of the 4.5-mas proper motion $(\sim 1 / 3$ of the synthesized beam FWHM), which takes place during each 12-hour synthesis. Subdivision of each observation was, however, not permitted by the data quality. The effects of this predictable motion have to be investigated more closely, but the lateral smearing that will result does not affect our conclusions about the existence of a brightening zone, derived from this sequence of independent maps. 
Discussion of the paper by $\underline{\text { R. DAVIS }}$ (p. 277)

Simon: Since you have both an angular expansion velocity and (presumably) an optical radial expansion velocity, can you say anything about the distance to this nova? Is the distance consistent with other distance estimates?

R. Davis: The distance is measured as $1.6 \mathrm{kpc}$, which gives an expansion velocity of $3800 \mathrm{~km} \mathrm{~s}^{-1}$. This velocity is consistent with the initial optical line widths. However, the line widths become narrower with time, but the expansion appears to continue to $3800 \mathrm{~km} \mathrm{~s}^{-1}$.

Molnar: Which of the three components in your map do you identify with the separate thermal and nonthermal components?

R. Davis: The thermal contribution to the map is calculated to be $0.1 \mathrm{mJy}$, and thus all the components are nonthermal. The thermal component presumably emerges in the central region.

Shapiro: Is it possible that your two ("double-sided") jets are a ring of material viewed edge-on?

R. Davis: Yes, indeed, A. R. Taylor is investigating just such a possibility. I am investigating an SS433 type of jet.

Discussion of the paper by MOLNAR (p. 279)

Schilizzi: Is there any evidence for changes in the orientation of the expanding components that might suggest precession such as observed in SS433?

Molnar: Not yet. For precession similar to SS433, one might expect the position angle to vary over a range of $\sim 40^{\circ}$. VLA and MERLIN measurements following giant flares in September 1982, September 1983, and October 1985 are all consistent with the same position angle ( $5^{\circ}$ errors on each measurement).

R. Spencer: We have made continuous observations of Cyg X-3 over a 60-hour period with the Jodrell Bank Mk1A-MkII short baseline interferometer at $\lambda 6 \mathrm{~cm}$, in August 1986. The most dominant period is 9 hours, with well separated flares. The individual flares last $\sim 4-6$ hours.

Molnar: As flare amplitudes vary from one to the next, the best way to search for the $4.95^{h}$ period is to look at the times at which each flare begins rather than at a Fourier transform of the entire data set.

Johnston: The flaring events in Cyg X-3 may be due to beaming. The orbital period of the binary system is $4.8^{h}$. How does one rationalize your $4.95^{h}$ period for flaring events with the orbital period if the variations that are called flares are due to beaming?

Molnar: The observed radio period, $4.95^{h}$, is not consistent with the orbital period, $4.79^{h}$. As the observed spectrum and VLBI size of flares both indicate that the 
physical size increases linearly with time since the beginning of a flare, it seems that the observed variation is due to a physical change of the source and not due to a change of the angle from which we view the source due to rotation. We therefore do not expect a priori to see the orbital period as we would from a geometrical model like beaming. The similarity of the radio period to the orbital period does suggest that the timing mechanism is connected to the orbital motion but mixed with some other frequency.

\section{Discussion of the paper by $\underline{\text { RICKETT (p. 287) }}$}

Diamond: With regard to your diagram of scattered size vs. galactic latitude, Kemball, Diamond, and Mantovani have new VLBI observations of $\mathrm{OH}$ masers at $1.66 \mathrm{GHz}$ that show that some masers at $|b|<2^{\circ}$ have sizes between 0.8 and 2.5 mas. Is this an embarrassment for the size vs. $|b|$ diagram?

Rickett: This result is interesting, and it appears to emphasize the wide disparity in level of scattering in lines of sight toward the galactic plane.

\section{Discussion of the paper by GWINN (p. 295)}

Ekers: Given the very large variations in scattering from source to source that were just discussed by Rickett, how do you justify the assumption of a large number of scattering centers?

Gwinn: The level of scattering varies widely in the Galactic Plane on scales of a degree or less; our results are consistent with uniform scattering over the extent of the cluster of maser features, a few arcseconds. Even if a single cloud is responsible for all the scattering, its extent along the line of sight should be sufficient to produce many independent deflections, yielding Gaussian statistics for the wander, if it is more than 10 A.U. deep, or about $10^{-4}$ its breadth.

\section{Discussion of the paper by PADRIELLI (p. 297)}

Ananthakrishnan: Did you say that you find the variability percentage falling as a function of latitude in the case of short time variable sources?

Padrielli: There is a tendency for the $m t$ products to be larger for galactic latitudes less than $20^{\circ}$. However, there are noticeable exceptions to the trend with the galactic latitude: DA406 and $1117+14$ (single time scale variables) vary too slowly or too much, and some sources with low galactic latitude in the direction of the galactic anticenter show $m t$ products much smaller than expected.

Fiedler: I would like to point out that DA406 displayed an extreme scattering event from 1985.3 to 1985.6. Do you have any data in this time frame?

Padrielli: We have monitored the source monthly until September 1985. The source shows a secondary peak of $\sim 0.3 \mathrm{Jy}$ in $\sim 1985.1$, and then it shows a monotonic decrease until the end of our observations. 
Discussion of the paper by STANDISH (p. 309)

Shapiro: It is also necessary to define a space-time geometry in order to make use of any direction prescription of a reference frame.

Burke: One hopes that radio astronomers will start to appreciate the advantages of giving positions referred to J2000 rather than B1950. No problems with source names (generally defined by position nowadays) arise if IAU conventions are adhered to.

Bartel: Did you find any time dependence of the rotation parameters you estimated for the relation between DE118 and PEP740R?

Standish: I did not explicitly solve for any. However, had I done so, the angular rotation about the $z$-axis would have reflected the $0.005 /$ cty shown in the difference of the (geocentric) solar mean motion in Table III.

\section{Discussion of the paper by JOHNSTON (p. 317)}

Preuss: Will it be possible in the framework of your program to measure the coincidence of a milliarcsecond radio feature with the nucleus of a bright Seyfert galaxy?

Johnston: This program is aiming at accuracies at optical wavelengths of $0 !^{\prime \prime} 03$. Thus, if the radio and optical reference frame can be aligned to this accuracy, then this is the accuracy of the coincidence. HIPPARCOS, if successful, will be accurate to $0 . \prime 003$. This should allow the coincidence of radio/optical features to be aligned on mas scales, again if the relationship of the optical and radio reference frames are defined at the mas level.

Cannon: In view of the fact that the quasars have very small proper motions (if any at all), do you have any estimate of the rate of degradation of the accuracy of the radio reference frame?

Johnston: This effect should be very small and has yet to be observed. Changes in source structure, if not monitored, will have a larger effect. I estimate this effect at the 0.1 mas level.

Morimoto: I have several comments: precession does not randomly change RA and Dec; the optical-radio reference frame tie must be heavily affected by differential rotation of the galaxy; at an accuracy of 0 ! $^{\prime} 01$, galactic rotation (not differential rotation) can be measured in $\sim 2-3$ years.

Hemenway: We are coming to the point where the ability to determine the differences between reference frames (e.g., radio and optical) will be limited by our knowledge of the structures and their differences (in frequencies and time) of the objects used to define the frames. In response to a previous question, I would like to add that in finding the relationship between a radio map and an optical map (images), if the radio map has absolute coordinates at the 0."01 level (from HIPPARCOS), then the Hubble Space Telescope may be used to point to 0.01 
accuracy if the guide stars are determined with that accuracy. A camera image would then be relatable to the radio image at the $0 !^{\prime \prime} 01$ or $0 .{ }^{\prime \prime} 02$ level.

Discussion of the paper by COATES (p. 337)

Preuss: In your diagram "accuracy vs. time (years)," there is a dotted line advancing into the $\mathrm{mm}$ accuracy regime for the next decade. Is this meant to be a (serious) goal of your operations?

Coates: Yes. NASA's goal of millimeter accuracy is based on the requirements of the scientists to get crustal motion velocities to an accuracy of $1 \mathrm{~mm} /$ year. This is discussed in T. Jordan's paper. It will take a lot of work to reach millimeter accuracies.

Chu: In your first photograph, What is the ring disk beneath your antenna? Is it for reducing the ground radiation?

Coates: It is for aerodynamic purposes.

Discussion of the paper by GORDON (p. 351)

Shapiro: What are the causes of the difference between the rate of motion of the Pacific with respect to the North American plate in your model and the RM2 models? Why is this difference apparently not covered by the error bar on this rate in the RM2 model?

Gordon: The difference in the direct estimate of the rate is probably because, until a few years ago, the magnetic data from the Gulf of California were too sparse to determine spreading rates reliably. The difference in the indirect rate reflects systematic differences between the NUVEL-1 and RM2 data sets along the East Pacific Rise. Part of the difference is caused by a revision of the geomagnetic reversal time scale a few years ago. The cause of the rest of the difference is unclear but might be due in part to slightly different time-averaging intervals between the two models.

Jordan: I have a comment on Shapiro's question. The differences between RM2 and NUVEL-1 Pacific-North America motions are evidently due to bias in the RM2 data set. It is interesting to note that this discrepancy, perhaps the largest between the two models, amounts to only about $8-10 \mathrm{~mm} /$ year, compared to a formal one-sigma uncertainty of $\pm 3 \mathrm{~mm} /$ year for RM2.

Sauber: In your abstract, you referred to variability in plate motions on time scales of $0.5-1$ million years. You did not discuss this in your talk; do you have any comments?

Gordon: Some changes of about $10 \%$ are seen. For the Pacific-North American plate motion, the rate based on recent data $(700,000)$ suggests a spreading rate of $52 \mathrm{~mm} /$ year vs. $48-50 \mathrm{~mm} /$ year (averaged over 3 million years). 
Discussion of the paper by KROGER (p. 365)

Whitney: Can you explain the commonality between the data sets and between your analysis and that of J. Sauber? At what point do the analyses become independent?

Kroger: Our data set spans the period January 1980 to May 1986. I believe that Sauber is using a data set spanning the period October 1982 through the early part of 1987 . Since there are very few measurements before October 1982, we differ in the measurements made after 1986, which are not included in our analysis.

Wright: Owens Valley seems to be used as the reference station for measurements in California. What is the evidence that OVRO is stationary with respect to the North America plate? In particular, note that if OVRO is moving south, then the discrepancy vector between the San Andreas fault motion and the model goes away, and that this discrepancy is exactly the motion of Hat Creek, presumably with respect to OVRO.

Kroger: The Owens Valley-Westford vector does not change.

Wright: The discrepancy vector is orthogonal to this vector; what are the errors on the orthogonal component?

Kroger: $\pm 1 \mathrm{~cm} /$ year.

Wright: This is the same magnitude as the discrepancy; I propose that Owens Valley may be moving south by $1 \mathrm{~cm} /$ year.

Discussion of the paper by J. DAVIS (p. 367)

R. Gordon: Is the very small motion $(-3 \pm 2 \mathrm{~mm} /$ year $)$ motion between Haystack and Mojave stations consistent or inconsistent with the large motion $(\sim 15 \mathrm{~mm} /$ year) we have heard about in other talks inferred between Mojave and other sites from presumed-stable North America?

J. Davis: Yes. The Goddard group gets $-3 \pm 3 \mathrm{~mm} /$ year using an independent set of experiments.

Clark: Measurements continue with Alaska and Ft. Davis.

Discussion of the paper by EUBANKS (p. 369)

Tang: 1) Since you usually use very long baselines, I suppose that the source structure effects play some role as an error source. 2) Did you see some rotation of the reference frame in your data analysis?

Eubanks: 1) I think you are probably right, although we hope that source structure effects will appear random and thus will be accounted for by the additive noise. 2) The reference frame clearly rotates with respect to the quasars. Since the effects 
of Earth rotation must be removed empirically, it is thus only possible to estimate relative rotations with this method.

Discussion of the paper by HERRING (p. 371)

Ulvestad: The $\mathbf{0 . 1}$ milliarcsecond errors that you quote for the nutation terms are very small. Could you comment on the possibility that systematic errors may be occurring at a larger level?

Herring: We have carried out a number of studies of this data set (for example, processing the data set as disjoint sets, and interleaved but independent sets) and have detected no changes that are inconsistent with the (66\% confidence interval) one-sigma standard deviation estimates. Also, the power-spectral density function shows no anomalous peaks.

Cappallo: Might not decade-timescale source structure changes make it difficult to estimate reliably long-period nutation terms? Also, as the source pool slowly changes, it seems as though there will be a source-frame random walk, with a similar effect.

Herring: These are problems that will need to be addressed as more data are obtained and as the accuracy of estimates of the nutation series coefficients improve. However, the effects of changing source structure can be corrected, although we do not currently do this.

Caporali: The power spectral densities you showed give information on the amplitudes of the various terms. Spectral analysis also gives the phases of each term. In addition to amplitude corrections, are there also terms that need to be corrected only in phase, or both in phase and amplitude?

Herring: When we estimate the corrections to the coefficients of the nutation series, we use the phase information. The deviations of the phase from the values predicted by the IAU nutation series are given by the out-of-phase components in Table 1 of this paper.

Discussion of the paper by WAHR (p. 381)

Gwinn: Morelli and Dziewonski use seismic techniques to obtain $Y_{2}^{0}$ core-mantle boundary topology consistent with our interpretation of the nutations, and sub-km errors.

Cappallo: Since the excitation spectrum of the 14-month Chandler wobble seems to have well-defined lines, it seems likely that the amplitudes of the driving terms may not be smooth across the Chandler response bandpass. Wouldn't this call into question the use of a smoothed half-width of the response in order to infer a characteristic decay time and thus a long-term $Q$ for the solid earth?

Wahr: Yes, results would be improved if the excitation source could be identified and its effects removed. 
Discussion of the paper by ROBERTSON (p. 391)

Popelar: Are there any plans to increase the frequency of observations to obtain better data for determination of diurnal nutation?

Robertson: We are attempting to arrange special UT1 observing sessions using Richmond and Bologna at a rate of four observing sessions per day.

Herring: The so-called "diurnal polar motion" is incorporated into our nutation determination.

Babcock: The IRIS data set is certainly very impressive, particularly the highfrequency UT1 data. But you mentioned that polar motion information is required in order to determine UT1 from single-baseline experiments. Would you comment on the source of polar motion information used for these daily UT1 measurements?

Robertson: The pole position and nutation data used to reduce the daily observing sessions was obtained by interpolating the IRIS values for those parameters.

R. Gordon: Could you comment on the accuracy of the determination from VLBI data of the secular trend in polar motion, which you discussed in your Scientific American article last year?

Robertson: The problem of determining a secular drift is not limited by the accuracy of the pole position determination but by the relatively brief $(\sim 5$ years $)$ duration of the observations. It is difficult to distinguish short-term trends from truly secular trends.

Roberts: You should try observing at random times each day, rather than at a given time each day. This will remove your aliasing problem and permit you to extract information at frequencies far beyond $1 / 2$ (minimum data spacing). This is described in Roberts, Lehar, and Dreher (A. J., 97, 968, 1987).

Robertson: We have some constraints on our observing times, but this is an idea that should definitely be investigated.

\section{Discussion of the paper by LOSITO (p. 411) (presented by Verrone)}

Herring: What was the $\gamma$ quantity in your polar motion differential equations?

Verrone: The $\gamma$ factor in the Free Polar Equation is related to $\gamma_{1}$ and $\gamma_{2}$, which are basically direction cosines of the $\bar{c}$ axis, axis of rotation of the core, relative to the reference frame of the mantle. $\gamma=\gamma_{1}+i \gamma_{2} . \bar{c}=\gamma_{1} \bar{x}_{1}+\gamma_{2} \bar{x}_{2}+\left(\gamma_{3}+1\right) \bar{x}_{3}$.

\section{Discussion of the paper by CAMPBELL (p. 427)}

Readhead: The spectral index is a safer criterion to use in trying to identify the stationary component than the flux density of individual components. There are a number of objects in which the core is not the brightest component. In addition, 
the relative brightness often changes between epochs.

Campbell: Yes, this is an important piece of information. In our best computations, we used the brightest component as a first viable approach, knowing that each source has its own particular problem. This matter can only be dealt with by close cooperation between astrophysicists and geodesists.

Ratner: Along the line of the previous comment (about using spectral indices rather than flux density as criterion to identify reference points in a brightness distribution), in the case of 3 C345 one cannot identify the stationary point with the brightest point in a map. This has not worked in the past.

Campbell: Yes, we know that before the final reprocessing of all geodetic data, we have to look at the structure history of each source. But here also lies the advantage of the proposed method: by using the regular geodetic VLBI campaigns, such as IRIS and CDP, we will have this structure history.

Marcaide: There may be cases where you will not be able to use either the spectral index as a guide to identify the stationary feature, as just pointed out by Readhead, nor the brightest component for it. An example is $4 \mathrm{C39.25.}$

Shapiro: The criteria for reference points in the quasar will perforce be complicated because the sources are. In particular, the "brightest" component can change identity because the "same" component does not necessarily remain brighter than the other component(s) in the source.

Potash: In correcting geodetic measurements for ionospheric effects, the low frequency ( $\mathrm{S}$ band) measurements factor in with a relatively low weight, about 1/13. The effect of $S$ band source structure is, however, somewhat more important than this low weight suggests. This occurs because $S$ band source structures tend to be larger than those at $\mathrm{X}$ band, and the magnitude of the source structure corrections scale, on average, with source size.

Eubanks: Could I conclude from your delay error plots that delay rate errors introduced from source structure effects will also be significant?

Campbell: We also compute the rate corrections, but the rate observations normally have a much smaller weight in geodetic solutions.

Tang: If you apply $\delta$-functions as the source model, it is not necessary to map the source. You can fit directly the $\delta$-functions to visibilities. However, we do see significant differences in estimations of source structure effect between using $\delta$-functions and a Gaussian model. Therefore, we apply the more precise model Gaussians - for our calculations.

Discussion of the paper by KARDASHEV (p. 433)

Romney: I think one of your slides mentioned, in Russian, a two-year lifetime for the spacecraft. Is that right? 
Kardashev: Yes, two years.

Shapiro: How is the phase integrity of the signals to be assured in RADIOASTRON?

Kardashev: The phase stability will be assured by accurate measurements of the orbit by a ground-based network. Also, one of the space-earth interferometer's antennas is outside the atmosphere/ionosphere, which disturbs the phase.

\section{Discussion of the paper by SCHILIZZI (p. 441)}

Marcaide: The dual polarization option in QUASAT must be certainly tagged to the decision to use, or not to use, an offset antenna (with direct illumination, that is, without subreflector) since instrumental cross-polarization is expected to be a problem.

Schilizzi: Catarzi and Tofani (these proceedings) suggest that polarization measurements with an offset antenna will in fact be possible.

Chu: It seems to me that the offset illumination will cause considerable trouble in polarization measurements. Also, the spurious polarization caused by offset illumination is difficult to compensate for. Since the ground VLBA antenna is $25 \mathrm{~m}$ in diameter, could one use an umbrella-type antenna to increase the diameter of the space antenna and the sensitivity of the interferometer pairs?

Schilizzi: For QUASAT, we are studying offset and center-fed inflatable antennas as well as deployable mesh antennas. Umbrella-type antennas are not presently under consideration. I should note that not all of the antennas on the ground are $25 \mathrm{~m}$ in diameter; we will certainly use the larger telescopes (for example, some elements of the EVN) as well to increase the sensitivity.

Biermann: In Germany, we have proposed a 50-m high frequency antenna included in the national report on the future of astronomy - to support space VLBI.

Schilizzi: Very good news!

Discussion of the paper by LINFIELD (p. 457)

Barthel: Did your Gaussian models agree with earth-based data obtained at higher frequency?

Linfield: We have not yet checked.

Bartel: I am worried that your values for the brightness temperature are modeldependent. If you had, for example, estimated the full-width of a uniform sphere model for the sources' brightness distribution rather than the FWHM of a Gaussian, your brightness temperature values would come down by a factor of $\sim 3$ and would drop at least in some cases below the magic temperature value of $10^{12} \mathrm{~K}$. 
Linfield: Although the fitted FWHM of a uniform sphere might be significantly different, the resulting brightness temperature would be only slightly different than for our Gaussian models. The formula for converting FWHM to $T_{B}$ depends on the type of source model.

Cannon: Is the inverse Compton brightness temperature limit of $10^{12} \mathrm{~K}$ so firmly established that you can say with confidence that a brightness temperature of $3 \times 10^{12}$ is a "strong" violation of this limit?

Linfield: Yes, inverse Compton losses vary as a large power of the temperature, and $3 \times 10^{12} \mathrm{~K}$ is excluded for the source rest frame.

\section{Discussion of the paper by $\underline{R O M N E Y}$ (p. 461)}

Wielebinski: Will the correlator be built in one stage or do you expect to do the work in sections?

Romney: A subset correlator of six or seven stations and two channels will be built first. This is intended to be adequate for thorough verification of correlator performance, as well as to support the first stand-alone operation of the VLBA. Expansion to the full 20-station 8-channel processor will then follow immediately.

Kaufmann: How will data from different antennas reach the central processing facility in Socorro? Are there any possible real time operations?

Romney: Recorded data tapes will be shipped from each station to the central processing facility by air or surface freight. A near-real-time correlation capability is planned but is intended only for technical verification, rather than scientific purposes.

Marscher: How will the multifrequency capability be implemented, i.e., how many frequencies will be able to be observed at once, and how rapidly will one be able to switch frequencies?

Romney: Simultaneous multifrequency observations will only be possible at $\mathrm{S}$ and $\mathrm{X}$ bands, unless one or more of the dual-frequency options, mentioned above, are implemented subsequently. Switching between frequencies (or dual-frequency pairs) is accomplished by rotating the subreflector and is expected to require on the order of 10 seconds.

Chu: For the purpose of site selection of the Chinese VLBI Network, we have calculated the $u-v$ coverage with the VLBA. It seems that the $u-v$ coverage with the previous D2 configuration is more uniform. (The coverage of short spacings cannot be seen in the $u-v$ diagram.)

Walker: The u-v coverage with the D2 array did not include many short spacings. The high value of short spacings was given more weight in the later configuration studies. The current VLBA configuration has better coverage of short spacings than array D2. Since the number of VLBA telescopes was fixed early in the project, improving short spacing coverage required some degradation of long spacing 
coverage. I suspect that you are seeing this effect.

Discussion of the paper by WAN (p. 475)

Shaffer: How and when can we apply for observing time on the Chinese array?

Wan: Please write to Dr. Wan Tongshan, Division of Radio Astronomy, Shanghai Observatory, Shanghai, People's Republic of China.

Discussion of the paper by NORRIS (p. 485)

Shapiro: What has been your experience with the phase stability when using fiber feeds for transmitting LO signals?

Norris: Experiments have failed to attain the required stability with fibers, and so we are distributing the local oscillators via coax cable and using the optical fibers for IF transmission only.

deBruyn: Do you plan to bring the "compact array" to a very small configuration when doing VLBI (to have an acceptably large fanbeam)?

Norris: We have not yet established a policy on this, but we are aware of the problem. Probably we will decide on the basis of individual experiments and whether a trade-off between sensitivity and field-of-view can be made. Certainly moving the elements to a small configuration will be possible when required.

Discussion of the paper by $\underline{Y E N}$ (p. 489)

Simon: What is the physical principle of your wavelength clock?

Yen: The local oscillator frequency and station delay are adjusted continuously to remove the station-dependent frequency offset and delay based on an assumed reference position on the sky and station location. The arrival time and frequency is thereby referred to a fictitious station at the center of the earth. Hence, no first-order fringe rate and delay tracking are required in the processor.

\section{Discussion of the paper by $\underline{A L E F}$ (p. 523)}

Shapiro: Just a historical comment: Our group first succeeded with elimination of fringe-phase ambiguities in switched observations between two sources about 0.5 apart about five years before we were lucky enough to find two sources that would be in all telescope beams simultaneously.

Alef: I agree. However, I am not aware of any phase-reference map before Marcaide 1982.

Bartel: What is the uncertainty of your determination of the separation between 
components in the two sources?

Alef: The error in the measurement of the separation is dominated by the errors in the source structure. Our estimate is better than 0.5 mas.

R. Davis: Can you say whether your phase stability is limited by spatial or temporal fluctuations in the atmosphere?

Alef: At a source separation of 0.5 , we are certainly limited by temporal fluctuations.

Discussion of the paper by $\underline{R O G E R S}$ (p. 533)

Treuhaft: You mentioned that the rms delay fluctuation is proportional to the square root of the air mass. This is only true at time scales less than a few hundred seconds. Beyond that, the rms fluctuation is approximately proportional to the air mass.

Rogers: I took the figure from your paper and the air mass dependence fit the square root fairly well.

Treuhaft: But that was given for 200 seconds only. For longer times, the fluctuation dependence on air mass increases.

Rogers: That's correct.

Readhead: At mm wavelengths, the atmospheric coherence time is $10 \mathrm{sec}$ in many cases. If the noise in closure phase measurements over each 10-second period is $\gtrsim 2 \pi$ radians, then we cannot use the closure phase determined from 10 -second coherent averages to determine the mean closure phase since, for low SNR, $2 \pi$ ambiguities in the closure phase bias the mean toward zero. This difficulty can be avoided by using the bispectrum $\Gamma^{3}\left(x_{1}, x_{2}\right)=\Gamma\left(x_{1}\right) \Gamma\left(x_{2}\right) \Gamma\left(-x_{1},-x_{2}\right)$ of each 10 -second period and averaging this. The argument of the bispectrum is the closure phase.

Rogers: We "vector" average the closure phase for many 10-second segments, which overcomes the problem of $2 \pi$ ambiguities and results in a SNR that continues to improve with the square root of the total averaging time. We do in fact use the "bispectrum" although we have called it the "vector" closure phase.

Discussion of the paper by ELGERED (p. 543)

Eubanks: What factors could give rise to a zenith troposphere bias in the WVR data?

Elgered: There are several factors that may be significant. One is instrumental errors, i.e., a bias in any or both of the WVR channels. Another factor, depending on how the algorithm relating WVR observables and the wet delay is derived, is uncertainty in the attenuation coefficients of water vapor or biases of independent calibration methods. In addition to this, since the "wet" and the "dry" delays 
cannot be separated in the estimation process, a bias in the zenith delay can also arise from possible errors in the pressure readings at the site. 